\title{
Bayesian Surface and Underwater Navigation
}

\author{
Rickard Karlsson and Fredrik Gustafsson, Member IEEE
}

\begin{abstract}
A common framework for surface and underwater (UW) map-aided navigation at sea is proposed, as a supplement to satellite navigation based on the global positioning system (GPS). The proposed Bayesian navigation method is based on information from a distance measuring equipment (DME) and information from databases. For the described system, the fundamental navigation performance expressed as the Cramér-Rao lower bound (CRLB) is analyzed and an analytic solution as a function of the position is derived. As a solution to the recursive Bayesian navigation problem, the particle filter is proposed. Two detailed examples of different navigation applications are discussed: surface navigation using a radar sensor and a sea chart and UW navigation using a sonar sensor and a depth database. In extensive Monte Carlo simulations the performance is shown to be close to the CRLB. The estimation performance for the surface navigation is in comparison with GPS performance. Experimental data is also successfully applied to the UW application.
\end{abstract}

\section{Index Terms}

Sea navigation, Recursive Bayesian estimation, Particle filter, Cramér-Rao lower bound.

\section{INTRODUCTION}

$\mathbf{M}$ ODERN sea navigation systems are often based on satellite information from the global positioning system (GPS). For critical navigation applications, this sensor cannot be the only positioning sensor. In military applications, an independent backup sensor insensitive to GPS jamming is preferable. Even for civil applications the robustness against jamming may constitute one of the main design issues in the future. In [1], [2] the problem of intentional or unintentional GPS jamming is discussed and alternative backup systems are strongly recommended. This is discussed further in [3] where both bathymetric and celestial methods are described as alternatives to GPS navigation. At sea, the satellite signal is often received without problem, since there is a free line-of-sight to several satellites. However, under severe weather conditions, such as ice-building on the antenna, or due to the landscape geometry, or a system failure, GPS signals may not be available. There are also applications where the GPS signal is not available at all, for instance in underwater (UW) navigation. Hence, there are many reasons to use an independent navigation system.

Many navigation systems rely on linear models or models that are nearly linear. If the system is approximately described by a Gaussian probability density function (pdf) or at least a unimodal density, then the extended Kalman filter (EKF), [4],

R. Karlsson is with the Department of Electrical Engineering, Linköping University, Linköping, Sweden (e-mail: rickard@isy.liu.se, phone:+46 13 281890).

F. Gustafsson is with the Department of Electrical Engineering, Linköping University, Linköping, Sweden (e-mail: fredrik@isy.liu.se, phone: +46 13 282706, fax: +46 13 282622).

EDICS: 2-ADPT Adaptive Systems and Filtering, 2-ESTM Estimation Theory and Applications. 


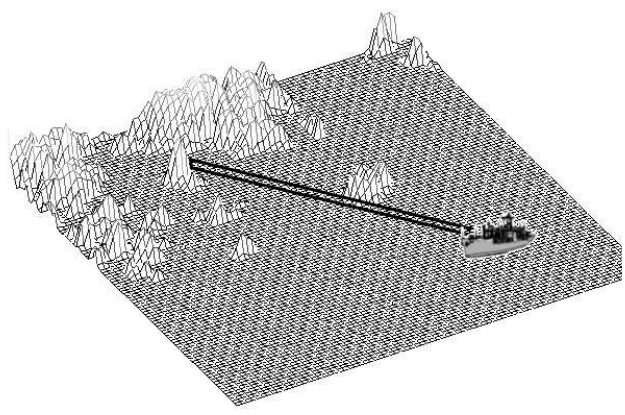

(a) Surface navigation with radar and a sea chart.

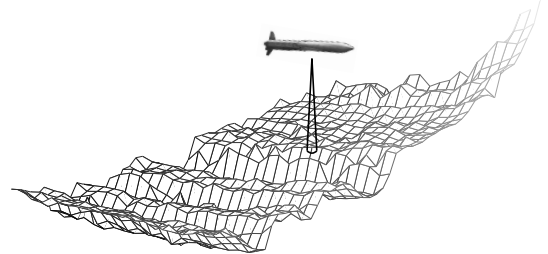

(b) UW navigation using a sonar and a depth map.

Fig. 1. Two DME navigation systems: (a) The surface navigation with a radar sensor and a sea chart database and (b) the UW navigation with a sonar sensor and a depth database.

[5], can often be applied successfully. For problems with highly non-linear or non-Gaussian distributions other methods must be used. One method is to use a bank of several Kalman filters. Several methods are available, for instance IMM, [6]. Also the Gaussian-sum filter, [4], which approximates the underlying pdf by several filters can be used. However, the most general approach is to tackle the non-linear and non-Gaussian problem directly using the particle filter (PF), [7], [8].

As an alternative to satellite navigation it is possible to use terrain information together with sensor information from a distance measuring equipment (DME). By comparing the terrain information from a database with the received DME measurements it is in many cases possible to get an accurate position estimate. This map-aided navigation technique is not a new topic, for instance in airborne navigation it has been applied frequently. In [9] a terrain-aided navigation is discussed using EKF; different linearization techniques and multiple models are discussed. In [10] six different stochastic linearization techniques are proposed. In [11], [12] a 3D matching technique using terrain maps is presented. In [13] the positioning is achieved using parallel filters. In [14] a Bayesian terrain navigation problem is solved using a numerical integration directly for the Bayesian recursions. In [15], [8] also a particle filter based method was proposed for aircraft terrain navigation. This was further investigated in [16]. For UW navigation mainly map-matching techniques or Kalman filter based techniques are previously used. See for instance [17], where a map-matching technique is described for positioning, and [18], where a Kalman filter and data association method is applied to sea floor map-aided navigation.

In this paper, the main focus is on the particle filter as the proposed solution to the recursive Bayesian navigation problem and on analysis of navigation performance, mainly using the Cramér-Rao Lower Bound (CRLB). A common framework for both surface and UW navigation using map-aided navigation together with DME information is formulated. A novel map-aided DME navigation system that does not require any external infrastructure and which is insensitive to jamming is proposed for surface navigation at sea. Using a radar sensor the range to shore in several directions can be compared to sea chart information, in order to calculate the probability for different locations. To the best of the authors knowledge, this is a completely new technique. Also a UW terrain-aided navigation using a sonar sensor is described, based on a similar technique as the airborne navigation system from [15] and using the preliminary results from [19], [20]. For the UW navigation a detailed depth map is compared to depth information retrieved from a sonar sensor in a similar way. For both applications range information from 
the DME and data from the movements, for instance speed and heading, are used to calculate the position. In a statistical framework the pdf for the ship's position is calculated. This can be achieved by considering several possible positions and for each one calculate the probability, using the particle filter. In Fig. 1 two navigation applications are illustrated.

The paper is organized as follows: In Section II the navigation system is described. In Section III its fundamental performance is discussed using the CRLB. In Section IV the Bayesian estimation problem is formulated and the approximate solution using the particle filter is described. In Section $\mathrm{V}$ the novel surface navigation method using a sea chart and a radar sensor is presented. Also the UW navigation based on sonar measurements and a depth database, [19], [20] is presented. Both extensive Monte Carlo simulations and experimental data are used. Finally, in Section VI conclusive remarks are given.

\section{NAVIGATION Models}

In this section the common model used in the surface navigation and UW navigation system is presented. Both the system dynamics and the measurement relation are discussed.

\section{A. Motion Model}

Depending on the configuration, different sensors can be used, such as speedometers and accelerometers. Hence, the motion can be modeled using as many position derivatives as desired. Here, only longitudinal and lateral motion is considered, where the speed is measured. Consider the following state variables: Cartesian position $(X, Y)$, and crab angle, $\delta$, that is the angle between the velocity vector and the stem of the ship,

$$
x_{t}=\left(\begin{array}{lll}
X_{t} & Y_{t} & \delta_{t}
\end{array}\right)^{T}
$$

as depicted in Fig. 2. The following discrete time model with sample time $T$ is used for the navigation system

$$
x_{t+1}=f\left(x_{t}, u_{t}, w_{t}\right)=\left(\begin{array}{c}
X_{t}+v_{t} T \sin \left(\varphi_{t}-\delta_{t}\right) \\
Y_{t}+v_{t} T \cos \left(\varphi_{t}-\delta_{t}\right) \\
\delta_{t}
\end{array}\right)+w_{t}
$$

with input signal $u_{t}=\left(\begin{array}{llll}v_{t} & \varphi_{t} & \theta_{t} & \phi_{t}\end{array}\right)^{T}$, consisting of speed, $v_{t}$, compass, $\varphi_{t}$, elevation angle, $\theta_{t}$ and azimuth angle, $\phi_{t}$ relative to the ship's stem. The sensor azimuth angle, $\phi_{t}$, and elevation angle, $\theta_{t}$, are not present in the dynamical model, but will be used in the measurement relation described in Section II-B. The process noise, $w_{t}$, is considered independent and describes the model uncertainty. Note that here, the known input signal is in practice values obtained from sensors. Hence, if they are considered as noisy measurements, the process noise will also describe the uncertainty in the input. The symbols used in the navigation model is listed in Table I. If $\delta$ is negligible or known the model simplifies even further to only position states. More advanced dynamical models can also be used, for instance a coordinated turn model, which was the case in [20]. For an overview of possible motion models, see the survey in [21]. 
TABLE I

DESCRIPTION OF SYMBOLS FOR THE NAVIGATION SYSTEM.

\begin{tabular}{|l|l|l|}
\hline Signal & Symbol & Description \\
\hline \hline States & $X_{t}$ & Position (in east direction) \\
& $Y_{t}$ & Position (in north direction) \\
& $\delta_{t}$ & Crab angle \\
\hline Inputs & $\varphi_{t}$ & Compass angle \\
& $v_{t}$ & Speed \\
& $\phi_{t}$ & Sensor azimuth angle relative to vessel \\
& $\theta_{t}$ & Sensor elevation relative to vessel \\
\hline Measurements & $r_{t}$ & Radar range or sonar depth \\
\hline Noise & $w_{t}$ & Process noise \\
& $e_{t}$ & Measurement noise \\
\hline
\end{tabular}

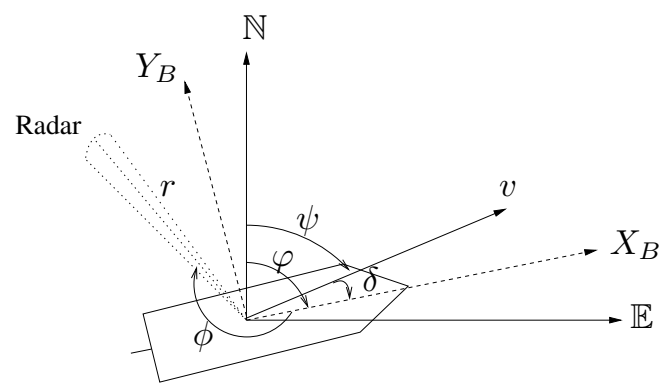

Fig. 2. The ship with compass angle $\varphi$ defined from the north direction $(\mathbb{N})$, together with the ship's body coordinate frame $\left(X_{B}, Y_{B}\right)$, crab angle $\delta$, velocity $v$ and heading $\psi$. The radar lobe is given with azimuth angle $\phi$ and the range $r=r\left(X, Y, \phi^{N}\right)$, where $\phi^{N}=\varphi+\phi$.

\section{B. Measurement Model}

For the surface navigation the range to land objects is measured by a radar sensor, and for the UW navigation the range to the sea floor is measured by a sonar sensor. Both sensors measure distance in the direction of the sensor. Hence, the measurement relation is given by

$$
y_{t}=h\left(x_{t}, u_{t}\right)+e_{t}=r\left(x_{t}, \phi_{t}^{N}, \theta_{t}\right)+e_{t}
$$

where $r\left(x_{t}, \phi_{t}^{N}, \theta_{t}\right)$ is the measured range from position $x_{t}$ and with the sensor azimuth angle, $\phi_{t}^{N}=\varphi_{t}+\phi_{t}$, relative to north and with elevation angle $\theta_{t}$. For the surface navigation the radar angle is defined in Fig. 2.

The DME cases for sea navigation is presented in Table II. Four different sensor types are discussed. The radar uses only azimuth information, hence $\theta_{t}=0$. The sonar system uses a fixed elevation. Also the GPS sensor can be viewed as a DME sensor, measuring range for a given position. Finally, the celestial sensor return a binary value. For all the cases different databases are used, for instance a sea chart database, a depth database, a satellite motion database and a star position database. 
TABLE II

Special cases of Distance Measuring Equipment (DME).

\begin{tabular}{|l|l|l|l|}
\hline Angles & Distance & Sensor & Database \\
\hline \hline$\theta_{t}=0$ & $r\left(x_{t}, \phi_{t}^{N}\right)$ & Radar & Sea chart \\
\hline$\theta_{t}<0$ & $r\left(x_{t}, \phi_{t}^{N}, \theta_{t}\right)$ & Sonar & Depth map \\
\hline$\theta_{t}>0$ & $r\left(x_{t}\right)$ & GPS & Satellite information \\
\hline$\theta_{t}>0$ & $r\left(x_{t}\right)$ & Celestial & Star information \\
\hline
\end{tabular}

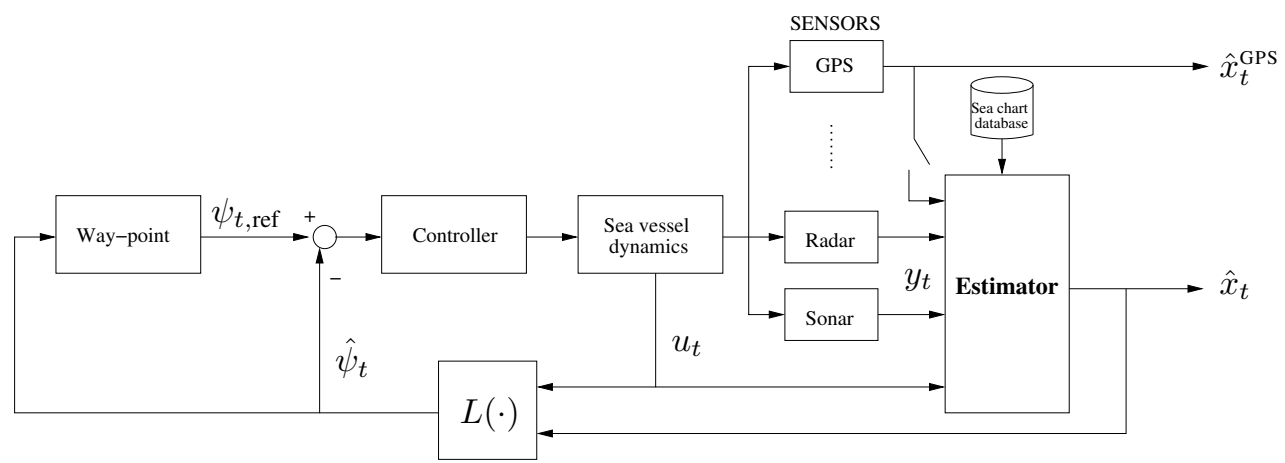

Fig. 3. The complete navigation system, used in the applications in Section V, consisting of a way-point unit, a controller, the sea chart database, the estimator and various sensors. The reference is given by the desired heading, $\psi_{t, \text { ref }}$ and the estimated heading is given as $\hat{\psi}_{t}=L\left(\hat{x}_{t}, u_{t}\right)$.

\section{Navigation System}

In Fig. 3 the complete surface or underwater navigation system, together with way-point calculation and auto pilot, is depicted. Depending on the application, one or more of the presented sensors are available. For surface navigation, the GPS signal can in many cases be the prime navigation sensor, where the estimate from the map-aided navigation method can be used to monitor performance. The main objective in this paper is to analyze the map-aided navigation method, hence the GPS part is not further discussed.

\section{THE CRAMÉR-RAO LOWER BOUND (CRLB)}

The main objective in this section is to find fundamental limits for the estimation performance expressed in terms of the system properties, for instance as a function of the measurement noise or the information available in the sea chart. The Cramér-Rao Lower Bound (CRLB) is such a characteristic for the second order moment [22]. This is done considering a static and dynamic case respectively. In the first case, only the measurement model is considered. In the second, the complete system dynamics is analyzed. The idea in this section is to perform a local analysis in each point in the sea chart and use that to analyze the global behavior.

In the sequel, the CRLB analysis is heavily based on expressions involving gradients of scalar functions or vector valued 
functions:

$$
\begin{aligned}
& \nabla_{x} g(x)=\left(\begin{array}{c}
\frac{\partial g}{\partial x_{1}} \\
\vdots \\
\frac{\partial g}{\partial x_{n}}
\end{array}\right), g: \mathbb{R}^{n} \mapsto \mathbb{R}, \\
& \nabla_{x} g^{T}(x)=\left(\begin{array}{ccc}
\frac{\partial g_{1}}{\partial x_{1}} & \cdots & \frac{\partial g_{m}}{\partial x_{1}} \\
\vdots & & \vdots \\
\frac{\partial g_{1}}{\partial x_{n}} & \cdots & \frac{\partial g_{m}}{\partial x_{n}}
\end{array}\right), g: \mathbb{R}^{n} \mapsto \mathbb{R}^{m} .
\end{aligned}
$$

Also, the Laplacian for $g(x, y)$ with $x \in \mathbb{R}^{n}, y \in \mathbb{R}^{m}$ is defined as

$$
\Delta_{y}^{x} g(x, y)=\nabla_{y}\left(\nabla_{x} g(x, y)\right)^{T}, g: \mathbb{R}^{n} \times \mathbb{R}^{m} \mapsto \mathbb{R}
$$

\section{A. Static CRLB}

For an unbiased estimator, $\mathbb{E}(\hat{x})=x$, the CRLB, [22], is given by

$$
\begin{aligned}
& \operatorname{Cov}(x-\hat{x})=\mathbb{E}\left((x-\hat{x})(x-\hat{x})^{T}\right) \geq J^{-1}(x), \\
& J(x)=\mathbb{E}\left(-\Delta_{x}^{x} \log p(y \mid x)\right),
\end{aligned}
$$

where $J(x)$ is the Fisher information matrix and $y$ the measurement. Also note that an equivalent representation of the information, [22], is

$$
J(x)=\mathbb{E}\left(\nabla_{x} \log p(y \mid x)\left(\nabla_{x} \log p(y \mid x)\right)^{T}\right) .
$$

Particularly, for $y=h(x)+e, e \in \mathcal{N}(0, R)$ the information is

$$
J(x)=H^{T}(x) R^{-1} H(x),
$$

where

$$
H^{T}(x)=\nabla_{x} h^{T}(x) .
$$

Above it is assumed that $J(x)$ is invertible. If not, a local analysis can be done by averaging information around $x$, in order to get a full rank information matrix. For the case with several independent measurements $y^{(i)}, i=1, \ldots, M$, this is usually not a problem. The information is then given by

$$
J(x)=\sum_{i=1}^{M} J^{(i)}(x),
$$

due to the additivity of information, assuming that $J^{(i)}$ is the information in measurement $i$. 


\section{B. Dynamic CRLB}

The theoretical posterior CRLB for a general dynamic system was derived in [23], [24], [15], [8]. The general state space model can be used to derive fundamental limits for navigation performance. In many applications, $\delta_{t}$ is small and can be discarded from the analysis, or it is known. Hence, for the CRLB analysis the following simplified model is used

$$
\begin{aligned}
x_{t+1} & =x_{t}+u_{t}+w_{t}, \\
y_{t} & =h\left(x_{t}, u_{t}\right)=r\left(x_{t}, \phi_{t}^{N}\right)+e_{t},
\end{aligned}
$$

where the horizontal position state vector, $x_{t} \in \mathbb{R}^{2}$, and the input signal, $u_{t}$, are defined as

$$
x_{t}=\left(\begin{array}{c}
X_{t} \\
Y_{t}
\end{array}\right), u_{t}=\left(\begin{array}{c}
v_{t} \sin \left(\varphi_{t}\right) \\
v_{t} \cos \left(\varphi_{t}\right)
\end{array}\right) \text {, }
$$

assuming independent additive process noise $w_{t}$. The observation relation consists of range measurements with measurement noise $e_{t}$. Using standard notations, consider independent noise sources, with variances $Q_{t}=\operatorname{Cov}\left(w_{t}\right)$ and $R_{t}=\operatorname{Cov}\left(e_{t}\right)$, the posterior CRLB for filtering can be written as, [15]

$$
P_{t+1 \mid t+1}^{-1}=Q_{t}^{-1}+J_{t+1}-S_{t}^{T}\left(P_{t \mid t}^{-1}+V_{t}\right)^{-1} S_{t}
$$

where

$$
\begin{aligned}
V_{t} & =\mathbb{E}\left(-\Delta_{x_{t}}^{x_{t}} \log p\left(x_{t+1} \mid x_{t}\right)\right), \\
S_{t} & =\mathbb{E}\left(-\Delta_{x_{t}}^{x_{t+1}} \log p\left(x_{t+1} \mid x_{t}\right)\right), \\
Q_{t}^{-1} & =\mathbb{E}\left(-\Delta_{x_{t+1}}^{x_{t+1}} \log p\left(x_{t+1} \mid x_{t}\right)\right), \\
J_{t} & =\mathbb{E}\left(-\Delta_{x_{t}}^{x_{t}} \log p\left(y_{t} \mid x_{t}\right)\right),
\end{aligned}
$$

where the bound is given by

$$
\operatorname{Cov}\left(x_{t}-\hat{x}_{t \mid t}\right)=\mathbb{E}\left(\left(x_{t}-\hat{x}_{t \mid t}\right)\left(x_{t}-\hat{x}_{t \mid t}\right)^{T}\right) \geq P_{t \mid t} .
$$

If the model in (11) is considered with Gaussian process noise, it gives $S_{t}=V_{t}=Q_{t}^{-1}$. Hence, given the true trajectory for the state vector the posterior bound can be calculated.

In order to analyze the performance, without performing any simulations, a local analysis of the dynamical system is conducted. The assumption is that the covariance should reach a stationary value, i.e., consider a point $x^{0}$ and assume $\bar{P}(x)=$ $P_{t+1 \mid t+1}(x)=P_{t \mid t}(x)$, for all $x$ such that $\left|x-x^{0}\right|<\epsilon$. Using this assumption in (13), it is possible to obtain an expression for $\bar{P}(x)$. This is done by applying the matrix inversion lemma

$$
(A+B C D)^{-1}=A^{-1}-A^{-1} B\left(D A^{-1} B+C^{-1}\right)^{-1} D A^{-1},
$$

using $A=Q, B=D=\mathcal{I}$ and $C=\bar{P}(x)$, where $\mathcal{I}$ is the identity matrix. This gives

$$
\bar{P}^{-1}(x)=(\bar{P}(x)+Q)^{-1}+J(x),
$$


Solving for $J(x)$ and applying the matrix inversion lemma again with $A=B=D=\bar{P}(x)$ and $C=Q^{-1}$ yield

$$
J(x)=\bar{P}^{-1}(x)-(\bar{P}(x)+Q)^{-1}=
$$

$$
\left(\bar{P}(x)+\bar{P}(x) Q^{-1} \bar{P}(x)\right)^{-1} .
$$

Hence, if $J$ is assumed invertible, then

$$
\bar{P}(x)+\bar{P}(x) Q^{-1} \bar{P}(x)-J(x)^{-1}=0 .
$$

Completing squares gives

$$
\left(\bar{P}(x) Q^{-1 / 2}+\frac{1}{2} Q^{1 / 2}\right)\left(Q^{-1 / 2} \bar{P}(x)+\frac{1}{2} Q^{1 / 2}\right)
$$$$
=J(x)^{-1}+\frac{1}{4} Q \text {. }
$$

Multiply with $Q^{-1 / 2}$ from left and right in the expression

$$
\left(Q^{-1 / 2} \bar{P}(x) Q^{-1 / 2}+\frac{1}{2} \mathcal{I}\right)\left(Q^{-1 / 2} \bar{P}(x) Q^{-1 / 2}+\frac{1}{2} \mathcal{I}\right)
$$

$$
=Q^{-1 / 2} J(x)^{-1} Q^{-1 / 2}+\frac{1}{4} \mathcal{I} .
$$

Since all matrices are symmetric, a unique matrix square root can be defined. Hence, solving for the symmetric and positive definite matrix, $\bar{P}(x)>0$, now yields

$$
Q^{-1 / 2} \bar{P}(x) Q^{-1 / 2}+\frac{1}{2} \mathcal{I}=\left(Q^{-1 / 2} J(x)^{-1} Q^{-1 / 2}+\frac{1}{4} \mathcal{I}\right)^{1 / 2} .
$$

Hence, the covariance $\bar{P}(x)$ is given as

$$
\bar{P}(x)=-\frac{1}{2} Q+Q^{1 / 2}\left(Q^{-1 / 2} J(x)^{-1} Q^{-1 / 2}+\frac{1}{4} \mathcal{I}\right)^{1 / 2} Q^{1 / 2} .
$$

One problem with the CRLB using the information form is that the Fisher information, $J$, may in general be singular. Often because the measurement sub-space dimension is much smaller than the state-space dimension. This can be handled using one of the following methods:

(i) Since a global analysis using local behavior is performed, it makes sense to average the information in a neighborhood i.e., $J(x)=\frac{1}{M} \sum_{k=1}^{M} J_{k}\left(x^{(i)}\right)$, where $x^{(i)}$ are picked around $x$, so $\left|x-x^{(i)}\right|<\epsilon$.

(ii) Introduce more measurements, so $J(x)=H^{T}(x) R^{-1} H(x)$ becomes invertible.

(iii) A regularization term can be added, basically meaning that a fictitious measurement with a large variance is introduced.

In order to understand the analytic CRLB expression in (23) assume that $J$ is invertible, and consider the special case $Q=q \mathcal{I}$ and $R=r \mathcal{I}$. The covariance $\bar{P}(x)$ is rewritten in order to perform a Taylor expansion:

$$
\bar{P}(x)=\left\{\begin{array}{l}
-\frac{q}{2} \mathcal{I}+\frac{q}{2}\left(\mathcal{I}+4 q^{-1} J(x)^{-1}\right)^{1 / 2}, \\
-\frac{q}{2} \mathcal{I}+q^{1 / 2} J^{-1 / 2}\left(\mathcal{I}+\frac{q}{4} J\right)^{1 / 2} .
\end{array}\right.
$$


Introduce the signal to noise ratio (SNR) as

$$
\mathrm{SNR} \triangleq q J=\frac{q}{r} H^{T} H
$$

Hence, for large or small SNR, a Taylor expansion yields

$$
\bar{P} \approx\left\{\begin{array}{l}
J^{-1}\left(\mathcal{I}-\mathrm{SNR}^{-1}\right) \\
q\left(-\frac{1}{2} \mathcal{I}+\mathrm{SNR}^{-1 / 2}+\frac{1}{8} \mathrm{SNR}^{1 / 2}-\frac{1}{32} \mathrm{SNR}^{3 / 2}\right) .
\end{array}\right.
$$

\section{RECURSIVE BAYESIAN ESTIMATION}

Navigation problems are often treated as Bayesian inference. The two map aided navigation methods described in Section II are described by nonlinear problems. Consider the following general state-space model

$$
\begin{gathered}
x_{t+1}=f\left(x_{t}, u_{t}, w_{t}\right), \\
y_{t}=h\left(x_{t}\right)+e_{t},
\end{gathered}
$$

where $x_{t} \in \mathbb{R}^{n}$ denotes the state of the system, $u_{t}$ the input signal and $y_{t}$ the observation at time $t$. The process noise $w_{t}$ and measurement noise $e_{t}$ are assumed independent with densities $p_{w_{t}}$ and $p_{e_{t}}$ respectively. Let $\mathbb{Y}_{t}=\left\{y_{i}\right\}_{i=0}^{t}$ be the set of observations until present time.

The Bayesian estimation problem is given by, [25],

$$
\begin{aligned}
p\left(x_{t+1} \mid \mathbb{Y}_{t}\right) & =\int_{\mathbb{R}^{n}} p\left(x_{t+1} \mid x_{t}\right) p\left(x_{t} \mid \mathbb{Y}_{t}\right) d x_{t} \\
p\left(x_{t} \mid \mathbb{Y}_{t}\right) & =\frac{p\left(y_{t} \mid x_{t}\right) p\left(x_{t} \mid \mathbb{Y}_{t-1}\right)}{p\left(y_{t} \mid \mathbb{Y}_{t-1}\right)}
\end{aligned}
$$

where $p\left(x_{t+1} \mid \mathbb{Y}_{t}\right)$ is the prediction density and $p\left(x_{t} \mid \mathbb{Y}_{t}\right)$ the filtering density. The problem is in general not analytically solvable. To solve the non-tractable Bayesian estimation problem in an on-line application without using linearization or Gaussian assumptions, sequential Monte Carlo methods, or particle filters (PF), can be used.

In this section a brief description of the particle filter theory is given. For more details see [15], [8], [7], [26]. The particle filter method provides an approximative Bayesian solution to (28) by approximating the probability density $p\left(x_{t} \mid \mathbb{Y}_{t}\right)$ by a large set of $N$ particles $\left\{x_{t}^{(i)}\right\}_{i=1}^{N}$, where each particle has an assigned relative weight, $\gamma_{t}^{(i)}$, such that all weights sum to unity. The location and weight of each particle reflect the value of the density in that region of the state space. The likelihood $p\left(y_{t} \mid x_{t}\right)$ is calculated from (27) yielding

$$
\gamma_{t}=p\left(y_{t} \mid x_{t}\right)=p_{e_{t}}\left(y_{t}-h\left(x_{t}\right)\right)
$$

By introducing a resampling step, as proposed in [7], problems with divergence can be handled. This is referred to as sampling importance resampling (SIR), and is summarized in Alg 1. 


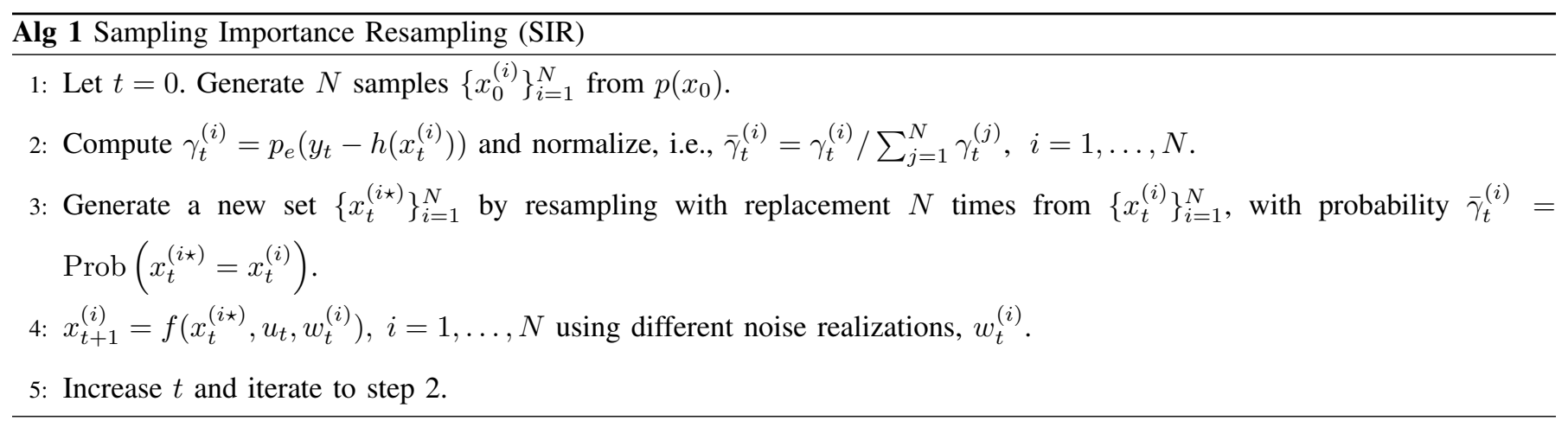

\section{ApPlications}

In this section, two specific applications of our general framework are presented:

- Surface navigation based on range information from the ship's radar and a sea chart.

- UW navigation based on sonar depth measurements and a detailed depth database.

For both applications Gaussian process noise and measurement noise are assumed. With more detailed motion models or sensor models other assumptions are possible.

\section{A. Surface Navigation}

In this section the novel navigation algorithm is described. The navigation system is presented in Fig. 3, where the ship is equipped with a radar, measuring relative distance to any land object. The sensor is assumed stabilized or that the deviation angle is small relative to the uncertainty in the radar sensor. To simplify the analysis, the speed relative to ground and the compass angle are considered as input signals, i.e., noiseless measurements, as described in Section II-A. However, this assumption is not necessary, and by introducing them as states-variables in the model, these can be estimated. Crucial for the positioning algorithm is a comparison of relative range measurements from the radar with expected land areas from a sea chart database. This is done in a statistically optimal way, using the particle filter as described in Section IV. The map presented in Fig. 4 is
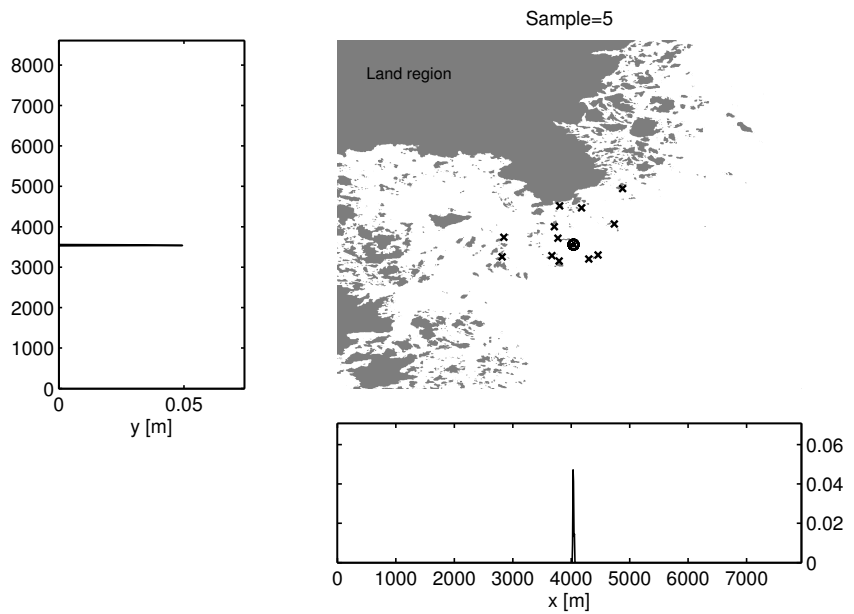

Fig. 4. The true position (o) and radar measurements (x), together with the particle cloud. The marginalized pdf for the $X$ and $Y$ directions are also shown. 
computed from a vectorized sea chart.

Since the speed is rather small compared to the radar revolution, a complete radar picture can be processed for each time the filter is updated, Fig. 5. In order to reduce the amount of possible range data, only a fixed number, $(M)$, of radar strobes are considered each revolution. The radar produces many range measurements in any given direction. In the algorithm, only the measurement closest to the ship is considered. The database consists of a sea chart, in the sequel a portion of the Baltic Sea is studied. In Fig. 4 the scenario is presented, where the ship's true position and some radar measurements are depicted. Also, the probability density function for each coordinate is given. As seen, after only 5 revolutions of the radar an accurate position is given. The initial distribution for the example given in Fig. 4 was uniformly distributed around the true position, $\pm 800 \mathrm{~m}$ in each direction. The radar measurements from Fig. 4 presented in a polar plot with the ship in the origin is presented in Fig. 5.

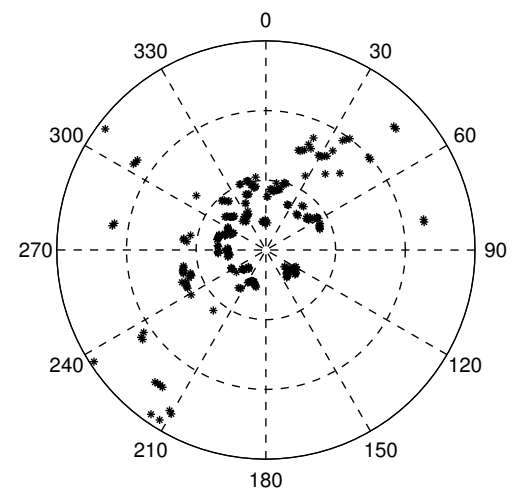

Fig. 5. The radar measurements from one revolution of the radar using a polar representation with the ship's position at the origin. The nearest distance to any object is visualized for all direction with the resolution given by the radar sensor.

In a Monte Carlo simulation study the scenario given previously is used. A straight trajectory is used in all simulations with different measurement noise realizations. In Fig. 6 the RMSE as a function of time is given for 50 Monte Carlo simulations. The parameters used in the Monte Carlo evaluation are presented in Table III. As seen in Fig. 6 the RMSE from the Monte Carlo simulations are close the fundamental limit. The performance using the map-aided navigation method equals that or is slightly better than of an ordinary GPS based navigation system, when there are sufficient returns from land objects. Also note that the peak is due to a region when very few of the radar strobes (from the downsampled radar picture) actually reflect any land area. Now $M$ different strobes are used each radar revolution, so even if land areas are present in the radar picture, sometimes no value is available in the downsampled set. Another possibility is that always $M$ different values are used. The theoretical CRLB calculation from Section III can be applied to the surface navigation problem. In Fig. 7 each point in the map is allocated the standard deviation $\operatorname{tr}(\bar{P}(x))$ using (23).

\section{B. Underwater Navigation}

In this section the UW navigation method from [19], [27] is described in detail, using the model from Section II. In [27] an UW terrain map was collected using sonar depth measurements and differential GPS. In Fig. 9 the original data is shown. 


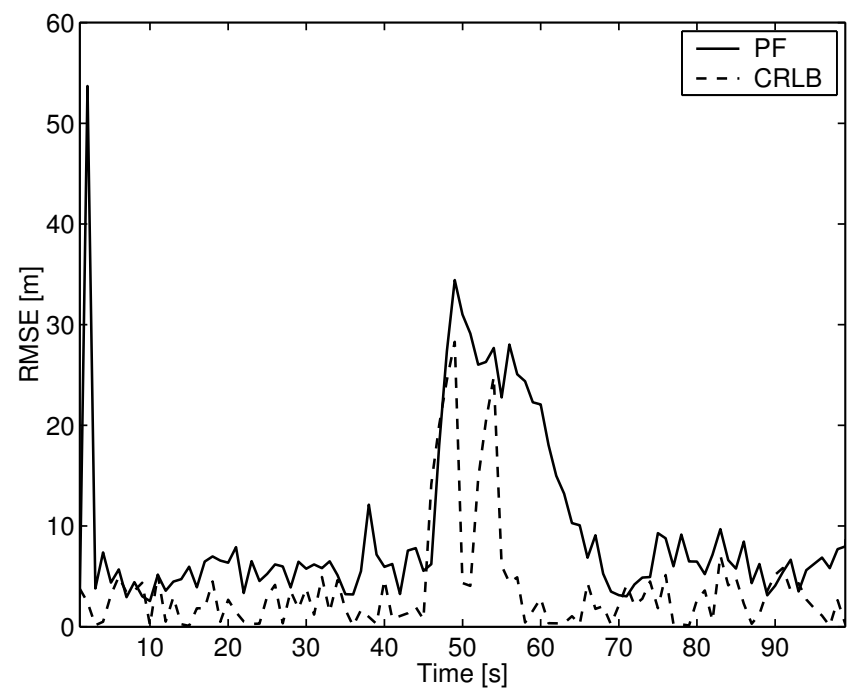

Fig. 6. Surface navigation position RMSE(t) for the particle from 50 Monte Carlo simulations together with the CRLB limit as the solution of the EKF around the true trajectory.

TABLE III

SURFACE NAVIGATION PARAMETERS.

\begin{tabular}{ll}
\hline \hline Monte Carlo simulations & 50 \\
Process noise covariance & $Q=\operatorname{diag}\left(10^{2}, 10^{2}, 0^{2}\right)$ \\
Measurement noise covariance & $R=10^{2}$ \\
Max radar meas./revolution & $M=16$ \\
Sample time & $T=1$ \\
Number of particles (initially) & $N=50000$ \\
Radar interval & $R_{\min }=300, R_{\max }=6000$ \\
\hline \hline
\end{tabular}

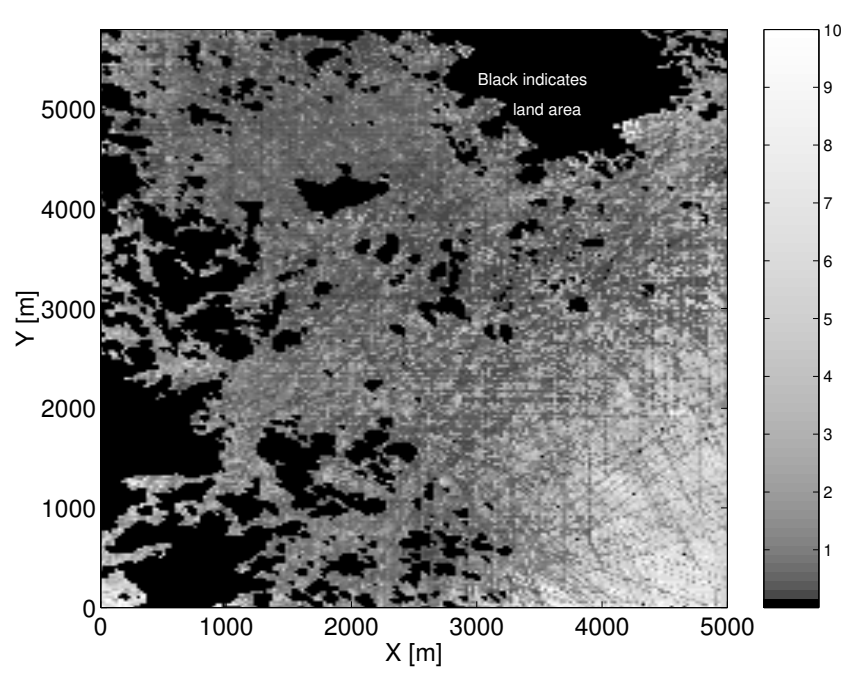

Fig. 7. The analytic CRLB for surface navigation for each position in the sea chart according to the analytic CRLB expression (23). 
This data is then interpolated and resampled to get a uniform depth map. This is depicted in Fig. 8, together with the platform at depth $d_{t}$ and with sonar range measurements $r_{t}$. In the experiment and in the simulations $d_{t}=0$. After the data for map

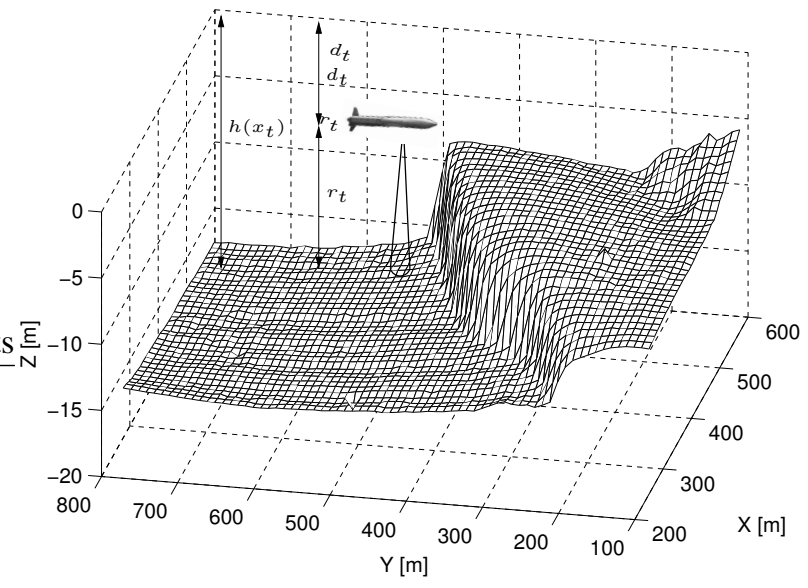

Fig. 8. UW navigation using sonar depth measurements and a true UW terrain database. The depth is $d_{t}$, and the sonar indicated the relative range to the sea floor, $r_{t}$. The database gives $h\left(x_{t}\right)$.

generation was collected, an independent test run in the map region was done, in order to collect measurements to test the map-aided navigation system. The experimental test run is presented in Fig. 10 together with the level curves. If the level curves are studied, one can see that the terrain is sufficiently varied for successful positioning even in the somewhat flat regions. In order to test the system performance, a Monte Carlo study on simulated data, but using the depth map from the

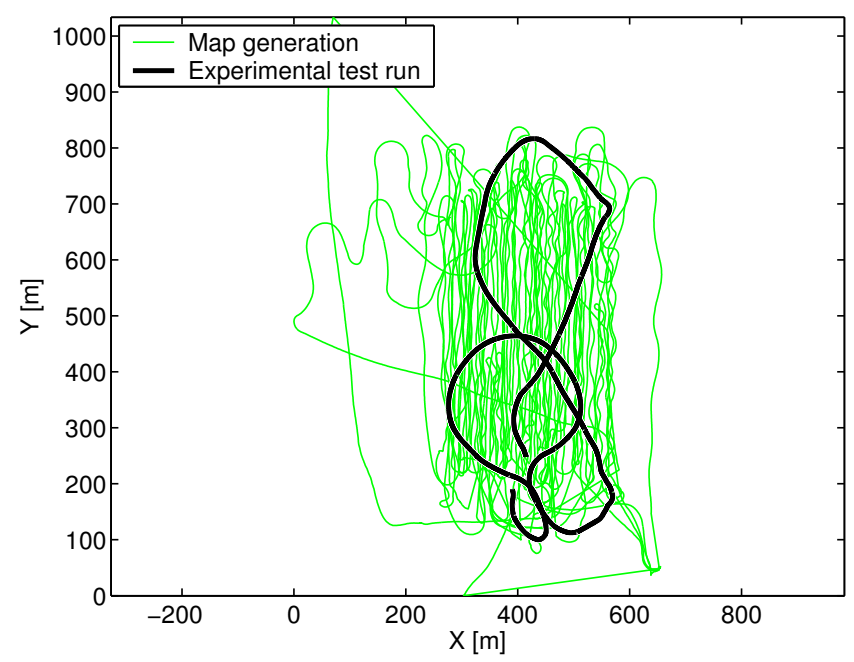

Fig. 9. The trajectory used to record depth data in order to produce the depth map, together with an independent test run on which the particle filter algorithm is tested.

experiment is made. The parameters used in the Monte Carlo evaluation and the experiment are presented in Table IV. The result is presented in Fig. 11. Note that in the upper plot, one experimental test run was used in the RMSE calculation. In the lower plot the RMSE is compared to the CRLB using 50 Monte Carlo simulations. The RMSE is close the the lower bound, and the error is mainly due to deficiencies in the map. 


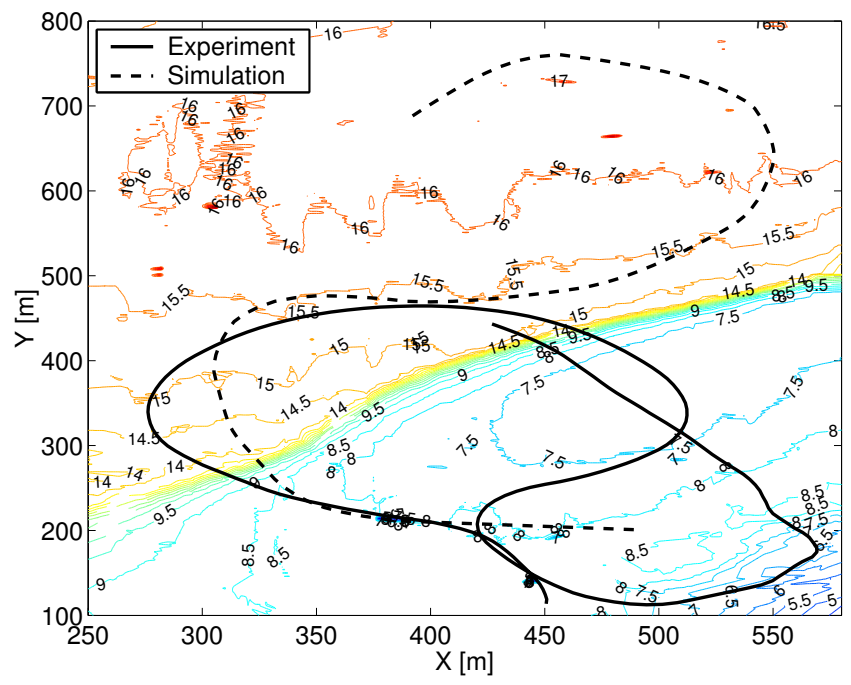

Fig. 10. Part of the experimental test run used for evaluation in the particle filter. The depth map is presented with level curves for each $0.5 \mathrm{~m}$ level. Also the trajectory used in the Monte Carlo simulation study is visualized.

TABLE IV

UW NAVIGATION PARAMETERS.

\begin{tabular}{ll}
\hline \hline Monte Carlo simulations & 50 \\
Process noise covariance & $Q=\operatorname{diag}\left(1^{2}, 1^{2}, 0^{2}\right)$ \\
Measurement noise covariance & $R=0.1^{2}$ \\
Sample time & $T=5$ \\
Number of particles (initially) & $N=50000$ \\
\hline \hline
\end{tabular}
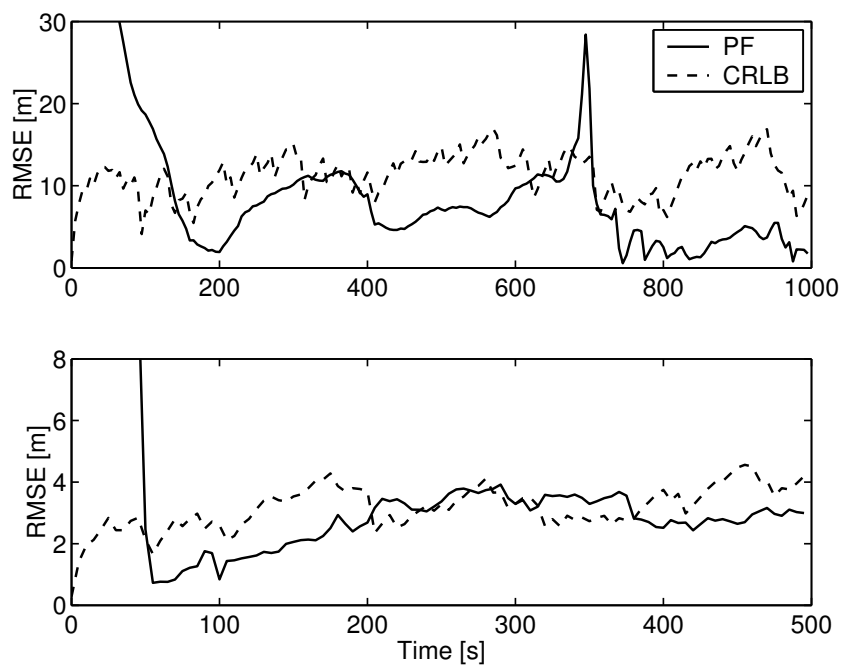

Fig. 11. Estimation error using the trajectories in Fig. 10. Upper: The position RMSE for the experimental test run and the CRLB as the EKF solution around the true trajectory. Note that only one test run was used in the RMSE calculation. Lower: The position RMSE from 50 Monte Carlo simulations. 


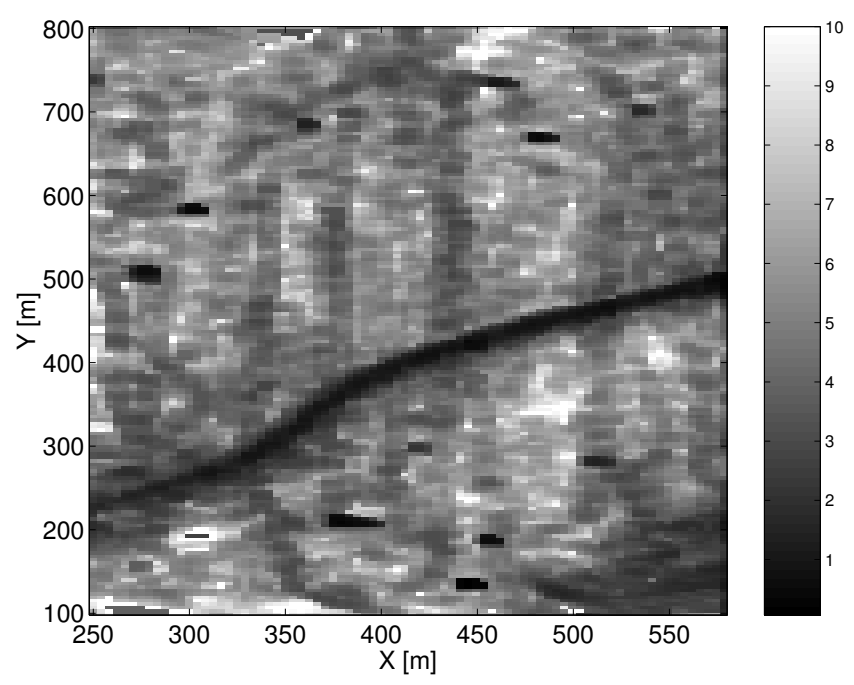

Fig. 12. The analytic CRLB according to (23), for each position in the map in Fig. 10.

In order to analyze the performance, without doing Monte Carlo simulations, the CRLB technique from Section III is used. The problem with the UW navigation is that only one depth measurement is given each time the filter is updated. Hence, the Fisher information, $J$, is singular, and the theoretical result cannot be applied directly. Since the idea behind the analytic CRLB was to use a local analysis of the dynamic system to get a global behavior, it is reasonable to average the information in a neighborhood of each point. Hence, the information matrix becomes invertible. The result from the CRLB calculations using (23) is presented in Fig. 12.

\section{CONClusions}

In this paper a framework for sea navigation using sea chart information and a distance measuring equipment is developed. The navigation performance is analyzed both theoretically and using Monte Carlo simulations. An analytic Cramér-Rao lower bound for the proposed navigation model has been derived. Two specific applications are studied in detail: surface and underwater navigation. The novel surface navigation method uses a radar sensor and a sea chart. In an extensive Monte Carlo simulation the system reaches GPS performance using only a radar sensor and the map-aided navigation method. The underwater navigation uses sonar and a depth map. Both Monte Carlo simulation and experimental data are successfully employed.

\section{ACKNOWLEDGMENT}

This work was supported by the VINNOVA's Center of Excellence ISIS (Information Systems for Industrial Control and Supervision) at Linköping University.

The authors would also like to thank: Saab Bofors Underwater Systems, Björn Johansson, Anna Falkenberg and Tobias Karlsson for providing UW topological data, and Björn Gabrielsson for initial discussions and valueful ideas. The Swedish Maritime Administration (Sjöfartsverket) for providing vector based sea chart information. Erik Svensson for assisting with sea chart vector data and map coordinate transformations. 


\section{REFERENCES}

[1] J. Volpe, "Vulnerability assessment of the transportation infrastructure relying on global positioning system final report," U.S. Department of Transportation. National Transportation Systems Center, Tech. Rep., Aug 2001. [Online]. Available: www.navcen.uscg.gov/archive/2001/Oct/ FinalReport-v4.6.pdf

[2] “GNSS Vulnerability \& Mitigation Measures. A Study for the European Maritime Radionavigation Forum (Rev. 6)," Tech. Rep., 2001.

[3] F. Pappalardi, S. Dunham, M. LeBlang, T. Jones, J. Bangert, and G. Kaplan, "Alternatives to GPS," in Proceedings of the MTS/IEEE Conference and Exhibition (OCEANS'01), vol. 3, Nov 2001, pp. 1452-1459.

[4] B. Anderson and J. Moore, Optimal Filtering. Englewood Cliffs, NJ: Prentice Hall, 1979.

[5] T. Kailath, A. Sayed, and B. Hassibi, Linear Estimation, ser. Information and System Sciences. Upper Saddle River, New Jersey: Prentice Hall, 2000.

[6] X. Li and Y. Bar-Shalom., "Design of an interactive multiple model algorithm for air traffic control tracking," IEEE Trans. on Control Systems Technology, vol. 1, pp. 186-194, 1993.

[7] N. Gordon, D. Salmond, and A. Smith, "A novel approach to nonlinear/non-Gaussian Bayesian state estimation," in IEE Proceedings on Radar and Signal Processing, vol. 140, 1993, pp. 107-113.

[8] A. Doucet, N. de Freitas, and N. Gordon, Eds., Sequential Monte Carlo Methods in Practice. Springer Verlag, 2001.

[9] L. Hostetler and R. Andreas, "Nonlinear Kalman filtering techniques for terrain-aided navigation," IEEE Transactions on Automatic Control, vol. 28, no. 3, pp. 315-323, Mar 1983.

[10] Y. Pei-Jun, C. Zhe, and J. Hung, "Performance evaluation of six terrain stochastic linearization techniques for tan," in Proceedings of the IEEE Aerospace and Electronics Conference, 1991, pp. $382-388$.

[11] J. Rodriguez and J. Aggarwal, "Navigation using image sequence analysis and 3-D terrain matching," in Proceedings on Interpretation of 3D Scenes, Workshop, 1989, pp. $200-207$.

[12] _ _ "Matching aerial images to 3-D terrain maps," IEEE Transactions on Pattern Analysis and Machine Intelligence, vol. 12, no. 12, pp. 1138 - 1149, Dec 1990 .

[13] P. Yubo, Z. Chen, and J. Hung, "BITAN-II: an improved terrain aided navigation algorithm," in Proceedings of the 1996 IEEE IECON 22nd International Conference on Industrial Electronics, Control, and Instrumentation, 1996, pp. 1675-1680.

[14] N. Bergman, Bayesian Inference in Terrain Navigation. Linköping Studies in Science and Technology. Thesis No 649, 1997.

[15] _ _ "Recursive Bayesian estimation: Navigation and tracking applications," Ph.D. dissertation, Linköping University, 1999, dissertations No. 579.

[16] M. Ahlström and M. Calais, "Bayesian terrain navigation with Monte Carlo method,” Department of Electrical Engineering, Linköping University, S-581 83 Linköping, Sweden, Master Thesis LiTH-ISY-EX-3051, 2000, in Swedish.

[17] L. Lucido, J. Opderbecke, V. Rigaud, R. Deriche, and Z. Zhang, "A terrain referenced underwater positioning using sonar bathymetric profiles and multiscale analysis," in Proceedings of the MTS/IEEE conference OCEANS '96, vol. 1, Sep 1996, pp. 417-421.

[18] D. di Massa and W. Stewart, "Terrain-relative navigation for autonomous underwater vehicles," in Proceedings of the MTS/IEEE Conference OCEANS '97, vol. 1, Oct 1997, pp. $541-546$.

[19] R. Karlsson, F. Gustafsson, and T. Karlsson, "Particle Filtering and Cramér-Rao Lower Bound for Underwater Navigation," in Proc. Acoustics, Speech, and Signal Processing, Hong Kong, Apr 2003.

[20] R. Karlsson and F. Gustafsson, "Particle filter for underwater terrain navigation," in IEEE statistical signal processing workshop, St. Loius, USA, Oct 2003.

[21] X. Li and V. Jilkov, "A survey of maneuvering target tracking: Dynamics models," in Proc. of SPIE Conf. on signal and data processing of small targets, Apr 2000

[22] S. Kay, Fundamentals of Statistical Signal Processing. Prentice Hall, 1993.

[23] H. Van Trees, Detection, Estimation and Modulation Theory. New York: Wiley, 1968.

[24] P. Tichavsky, P. Muravchik, and A. Nehorai, "Posterior Cramér-Rao bounds for discrete-time nonlinear filtering," IEEE Transactions on Signal Processing, vol. 46, no. 5, pp. 1386-1396, 1998.

[25] A. Jazwinski, Stochastic processes and filtering theory, ser. Mathematics in Science and Engineering. Academic Press, 1970 , vol. 64.

[26] R. Karlsson, Simulation Based Methods for Target Tracking. Department of Electrical Engineering, Linköping University, Sweden: Licentiate Thesis no. 930, Feb 2002. 
[27] T. Karlsson, “Terrain Aided Underwater Navigation using Bayesian Statistics," Masters Thesis LiTH-ISY-EX-3292, Department of Electrical Engineering, Linköping University, 2003. 